\title{
The Optimal Artificial Neural Network for Small Datasets on Shear Resistance Prediction of Steel Fibre Reinforced Concrete Beam
}

\author{
S. F. Senin", R. Rohim, A. Yusuff \\ School of Civil Engineering, College of Engineering, Universiti Teknologi MARA, Cawangan Pulau Pinang, 13500, \\ Permatang Pauh, Penang, Malaysia
}

Received May 7, 2021; Revised October 19, 2021; Accepted November 22, 2021

Cite This Paper in the following Citation Styles

(a): [1] S. F. Senin, R. Rohim, A. Yusuff, "The Optimal Artificial Neural Network for Small Datasets on Shear Resistance Prediction of Steel Fibre Reinforced Concrete Beam," Civil Engineering and Architecture, Vol. 10, No. 1, pp. 323-333, 2022. DOI: 10.13189/cea.2022.100127.

(b): S. F. Senin, R.Rohim, A.Yusuff (2022). The Optimal Artificial Neural Network for Small Datasets on Shear Resistance Prediction of Steel Fibre Reinforced Concrete Beam. Civil Engineering and Architecture, 10(1), 323-333. DOI: $10.13189 /$ cea.2022.100127.

Copyright $(2022$ by authors, all rights reserved. Authors agree that this article remains permanently open access under the terms of the Creative Commons Attribution License 4.0 International License

\begin{abstract}
This study presents an approach for the prediction of the shear strength of steel fiber reinforced concrete (SFRC) beams using the Artificial Neural Network (ANN) developed based on existing experimental shear resistance results from various researchers. The experimental results database containing 42 sample numbers of SFRC beams (with shear span-to-depth ratio exceeding 2.5) without stirrups, with compressive strength of concrete varying from 24.9 to $68.6 \mathrm{MPa}$ and steel fibers of hooked end type are used to develop an ANN model. The developed ANN model is trained by using $70 \%$ and $90 \%$ of the data and another 30 to $10 \%$ served as the validation data purpose. The shear strengths prediction based on ANN model was found to be in perfect agreement with the experimental values when the optimal neuron number is 2 and by fixing the training set size as $90 \%$. Results showed that this ANN model has strong potential as a feasible design tool for predicting the shear strength of SFRC beams without transverse reinforcement or stirrups within the range of input parameters considered in this study.
\end{abstract}

Keywords ANN, SFRC, Optimal Network, Shear Resistance

\section{Introduction}

Plain concrete, being a brittle material, cracks when exposed to low tensile stresses caused by shear stresses at inclined portions of a beam [1]. This failure normally results in devastating damage, both human and material, since it occurs unexpectedly and without notice, especially in reinforced concrete beams. The addition of steel fibres to concrete improves this tensile stress threshold value at the post-cracking level, thus increasing the overall shear resistance of SFRC beams [2]. Since the mechanisms of this shear resistance are still unknown, it is important to investigate the importance factors such as steel fibre -reinforced concrete (SFRC) volume, the dimension of beam geometry, the aggregate size, the compression strength of concrete to the overall shear resistance. Several model expressions are available in the literature [3-6] and existing codes [7] by expensive laboratory testing of full-scale beams have been developed, and these expressions are often semi-empirical, which has certain limitations in its accuracies. Due to this issue, there is a need to establish a computational solution that can be used to model shear resistance in SFRC due to a lack of simple equations in that describe shear resistance for fibre reinforced concrete (SFRC) beams.

Recently, machine learning approaches have been successfully employed to model the highly non-linear 
shear resistance in SFRC beam. In this regards, Nonlinear Regression Algorithm (NGA), Surface Response Methodology (RSM) and the Artificial Neural Network (ANN) were recently applied to predict the shear resistance of SFRC structure. NGA is considered as an important tool that is able to model the complex process in various engineering disciplines. However, the proposed model on predicting shear resistance based on NGA is less accurate than the ANN [8]. Works from reference [9-10] had concluded that the performance of data prediction by RSM is slightly less accurate than ANN. On the other hand, ANN is the most widely used and successfully in its prediction in civil engineering domain, despite its inability to produce practical prediction equations. ANN algorithm showed no assumption or hypothesis on the data distribution before using for modelling as compared to the above-mentioned algorithms. The more comprehensive on ANN methodology will be discussed more in section 2.1 in this article.

One of the challenges faced by the researchers in ANN modelling is the determination of the number of hidden layer numbers and its neurons numbers [11]. This is very important as it will give significant impact on the final outputs. A balanced selection between the hidden layers and the neuron numbers in each layer therefore must be carefully decided. Employing too few neurons in hidden layer will cause underfitting, a situation where it cannot capture the underlying trend of data [12]. On the other hand, using too many neurons in the hidden layer will result in increased in computational time and overfitting [13]. There are several articles as proof that network with single hidden layer is sufficient for universal approximations, and further supported by easier to execute and train by the network. 13-15]. Guogiang[16] had successfully employed 1 hidden layer with optimal number of neurons to predict the compression strength of high performance self-compacting concrete with correlation coefficient of 0.979. Liang Jia Lei [17] attempted to use 1 hidden layer with 16 neurons for the prediction of shear characteristics of unsaturated soil based on back propagation neural network. Kiran Shusama

[18] used 1 hidden layer with 20 neurons to forecast the soil shear strength using ANN.

In this paper, the ANN model development, and the prediction of SRFC shear resistance will be the conducted based on 1 hidden layer only to minimize the computational time usage. This paper explains the optimization the ANN performance of a single hidden layer network trained with the Leverberg-Marquard algorithm. The parameters relevant to ANN training as well as the design parameters are considered when developing the optimal ANN model. To demonstrate the methodology and its results, a case study on a small dataset of 42 shear resistance datasets is introduced.

\section{Materials and Methods}

\subsection{Datasets}

In this research, the datasets used were the results of experimental works of forty-two SFRC beams that were compiled from nine different independent authors (Amin \& Foster[19] ; Amin et al.[20] ; Greenough \& Nehdi[21] ; Hwang et al. [22]; Imam et al., [20]; Kwak et al., [24]; Qissab \& Salman,[25]; B. Singh \& Jain, [26]; Spinella, [27]; Zarrinpour \& Chao,[28]). The statistics information of the datasets is shown in Table 1 and all of them having shear span to depth ratio more than 2.5 , to ensure shear dominates the failure mechanism. The compiled datasets were consisting of the following parameters:

(a) bw (Width of web)

(b) $\mathrm{h}$ (Height of beam)

(c) d (Depth of beam)

(d) fck (Compressive strength)

(e) $\mathrm{Vf}$ (Volume of steel fiber in \%)

(f) da (Maximum aggregate size)

(g) Vutot (Experimental shear capacity)

$$
\begin{gathered}
X^{T}=\left[\begin{array}{llllll}
b_{w} & h & d & f_{c k} & V_{f} & d_{a}
\end{array}\right] \\
Y_{A}=\left[\begin{array}{ll}
V_{t o t}
\end{array}\right]
\end{gathered}
$$

\subsection{The Output Datasets Transformation and Input Datasets Normalization}

Data normalization is essential as the input vectors, $\mathrm{X}$, has varying scales and the statistical distribution of the input vectors data is unknown. This datum is scaled within the range of 0 to 1 by using the following data transformation algorithm.

$$
X^{\prime}=\frac{X-\min (X)}{\max (X)-\min (X)}
$$

where $\mathrm{X}^{\prime}$ is the normalized input vector, $\mathrm{X}$ is the original input vector, $\min (\mathrm{X})$ and $\max (\mathrm{X})$ is the minimum values and the maximum values of the original input vector, respectively.

As the output vector, $Y_{A}$, is a continuous outcome, and to ensure the entire data will exhibit nearly symmetric bell-shaped distribution, log-normal transformation is used to the transformed this skewed data to approximately conform to normality [29]. The log-normal transformation was done by using the following algorithm.

$$
Y^{\prime}=\log _{10}\left(1+Y_{A}\right)
$$


Table 1. Ranges of Parameters in Database

\begin{tabular}{ccc}
\hline Parameter (units) & Min & Max \\
\hline bw (mm) & 100 & 150 \\
h (mm) & 150 & 300 \\
d (mm) & 135 & 251 \\
fck (MPa) & 24.9 & 68.6 \\
Vf (\%) & 0.5 & 1.0 \\
da (mm) & 5 & 20 \\
Vutot (kN) & 900 & 2400 \\
\hline
\end{tabular}

\subsection{ANN Methodology}

After the data transformation, ANN must be setup for the training of the processed data. A single-layer feed forward network is made up of layers of neurons that can only pass in one way without any feedback. The Backpropagation (Bp) algorithm, which uses the gradient descent optimization approach, is the most often used method for training feedforward ANNs. $\mathrm{Bp}$ is a supervised learning approach that is used to train NN and is iteratively used to learn data.

In Figure 1, each normalized input, X', in the input layer is multiplied by a connection weight, wij, between the neuron $i$ in the input layer to the neuron $j$ in the first hidden layer. The product of this parameters will be summed as describe by Equation 5 and then passed to the output layer by a nonlinear sigmoid activation function in Eq 6 to gain the predicted output $V_{\text {utot }}$. in Equation 7.

$$
\begin{gathered}
I_{j}=\sum_{i=1}^{j} X_{j}^{\prime} w_{i j}+b_{j} \\
f(I)=\frac{1}{1+e^{-I_{j}}} \\
y_{j}=f\left(I_{j}\right)
\end{gathered}
$$

where $b_{j}$ is the bias value for updating the weights

It sends the signal $y_{j}$ from the first hidden layer to the neuron in the output layer and evaluate the input to the neuron in the output layer as in Eq 8. In this case, $\mathrm{k}$ is set as 1 as the output neuron number is one.

$$
V_{U T O T}=\sum_{j=1}^{k} y_{j} w_{i j}^{\prime}+b_{k}^{\prime}
$$

This ANN algorithm was created by using the MATLAB software.

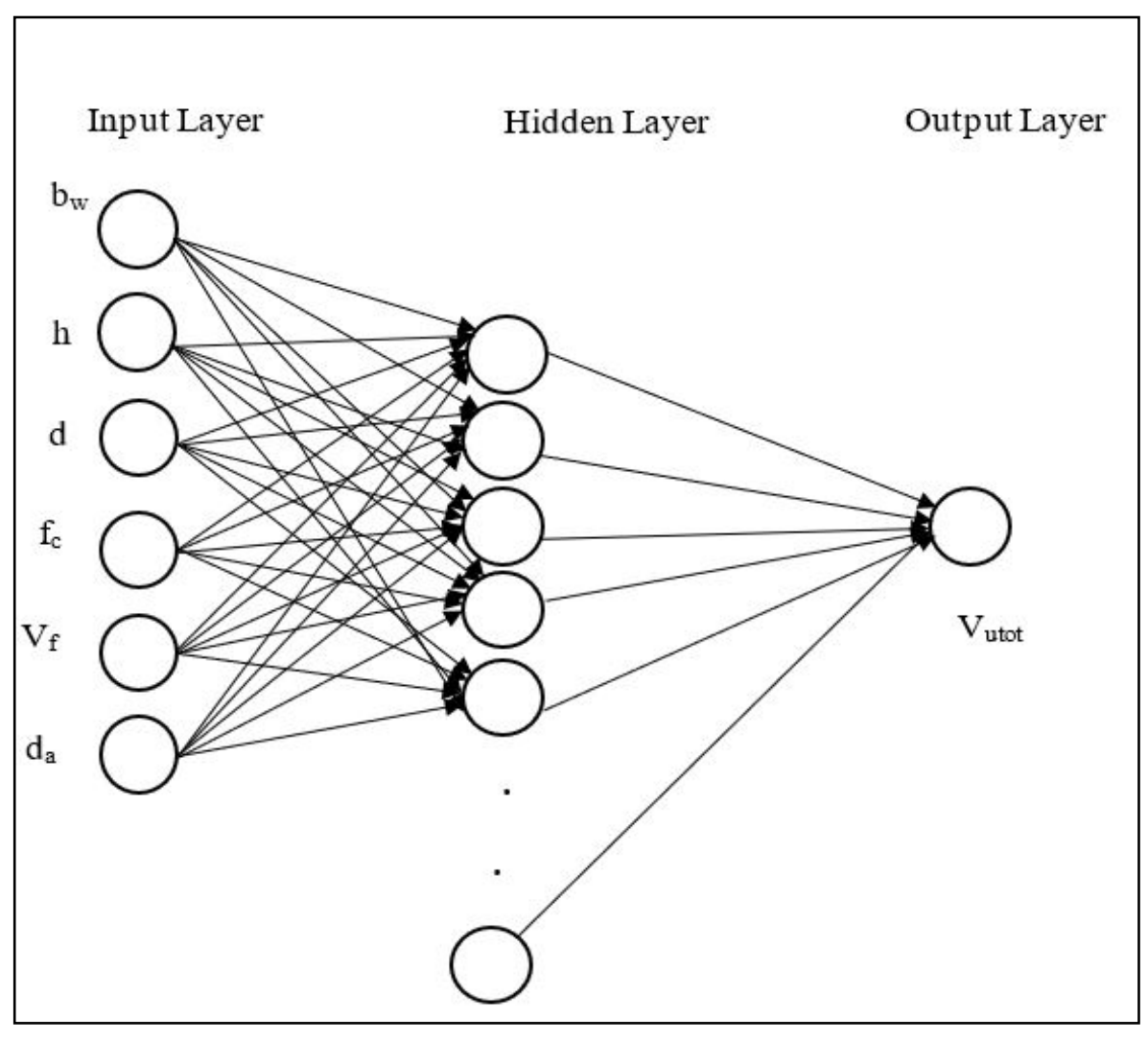

Figure 1. ANN architecture 
To minimize the time of computation for modelling, only 1 hidden layer was used in this study. In order to determine the optimum number of hidden neurons in the hidden layer, the performance metric of the ANN was investigated by varying the neurons numbers from 1 to 12 . The performance metric of the ANN model is characterized by the Root of Mean Square Error (RMSE) and coefficient of correlation (R) are given as follows.

$$
\begin{gathered}
R M S E=\sqrt{\sum_{i=1}^{N} \frac{\left(Y_{P}^{i}-Y_{A}^{i}\right)^{2}}{N}} \\
R=\frac{\sum_{i=1}^{N}\left(Y_{P}^{i}-\overline{Y_{P}}\right)\left(Y_{A}^{i}-\overline{Y_{A}}\right)}{\sqrt{\sum_{i=1}^{N}\left(Y_{P}^{i}-\overline{Y_{P}}\right)^{2}\left(Y_{A}^{i}-\overline{Y_{A}}\right)^{2}}}
\end{gathered}
$$

where $Y_{P}^{i}$ is the predicted shear resistance, $Y_{A}^{i}$ is the actual shear resistance from datasets, and $\mathrm{N}$ is the number of datasets. The symbol with hat symbol on each variable $Y_{P}$ and $Y_{A}$ is the mean value of the predicted and the actual shear resistance, respectively.

To ensure a more reliable ANN model, the datasets were divided into training, validation, and testing subsets. 70 percent and 90 percent of the dataset were considered for network training and the remaining datasets will be divided equally for network reliability and to avoid overfitting. The percentages of datasets for training, validation and testing are varied as given in Table 2 . There were no fixed rule on deciding the proportion of dataset sizes; but in majority of the reviewed papers, the training datasets were divided from $50 \%$ to $98 \%$. Most of the literatures on ANN modelling works attempted to use $70 \%$ [30-35] and 90\% [36-39] as the trial percentage training datasets and yields acceptable prediction capabilities. It is, therefore, a trial proportion value of $70 \%$ and $90 \%$ of the overall data had been chosen as the training data proportion division to measure both capabilities to predict the ultimate shear capacity of the beam.

Table 2. Percentage of Training, Validation and Testing Datasets

\begin{tabular}{|c|c|c|c|}
\hline Datasets No & Training & Validation & Testing \\
\hline 1 & 70 & 15 & 15 \\
\hline 2 & 90 & 5 & 5 \\
\hline
\end{tabular}

The optimal number of neurons of the hidden layer is determined by monitoring the RMSE values for training, validation, and testing datasets by the early stopping algorithm [40].

The convergence of the trained ANN model during training cycle is based on minimizing the root of mean square error (RMSE) value. The overall performance of the trained network is monitored by comparing the predicted shear resistance with the actual output from datasets in Table 1. The properties of the trained ANN are shown in Table 3.

Table 3. Properties of ANN Model

\begin{tabular}{|c|c|c|}
\hline Parameters & $\begin{array}{c}\text { Description of } \\
\text { parameters }\end{array}$ & Remarks \\
\hline Architecture & 6-(1 to $\left.12^{*}\right)-1$ & $\begin{array}{c}* \text { The optimal number } \\
\text { of neuron is } \\
\text { determined by early } \\
\text { stopping algorithm. }\end{array}$ \\
\hline $\begin{array}{c}\text { Performance } \\
\text { function }\end{array}$ & RMSE & $\begin{array}{c}\text { Root Mean Square } \\
\text { Error }\end{array}$ \\
\hline $\begin{array}{c}\text { Learning } \\
\text { Algorithm }\end{array}$ & Levenberg-Marquardt & Tansig \\
\hline $\begin{array}{c}\text { Activation } \\
\text { Function }\end{array}$ & $\begin{array}{c}\text { Non-linear mapping } \\
\text { function }\end{array}$ \\
\hline
\end{tabular}

\section{Results and Discussions}

\subsection{Skewness Analysis on the Dataset}

There is a great variety of possible ways of data transformation, from adding constant to multiplying, squaring, or converting to logarithmic scales etc. to transform the values of the shear resistance data, $\mathrm{Y}_{\mathrm{A}}$, to a symmetric distribution. The sources of such extreme values of shear resistance may possibly be contributed from the measurement errors from the equipment, the nature of the distribution variation and the way of sampling. Log-normal transformation is employed to the original shear resistance data to reduce its large variation of the data.

The skewness of the original output datasets, $Y_{A}$ was analyzed in the first place. The skewness of the original output dataset is 0.8442 , showing the presence of larger extreme values of shear resistance in the actual output datasets that distort the symmetric distribution [41]. By using the log-normal transformation on the actual output data as given in Equation 4, the skewness value of the transformed data, $Y^{\prime}$ is reduced from 0.8442 to 0.0361 , which is almost close to 0 . This indicates that the transformed output datasets are almost nearly fit to the normal distribution, which is required in many statistical models. Even though this is not a priori requirement to the neural network modelling development, but this practice may help the training process of the ANN model. Figure $2 \mathrm{~A}$ and Figure 2B show both the data distribution of the original and the transformed output data.

The original input vectors, $\mathrm{X}$ was normalized into range of 0 to 1 value only by using Equation 3 and the normalized input vectors, $X$ ' and the transformed output $Y^{\prime}$ will be used for modelling the shear resistance using ANN. 


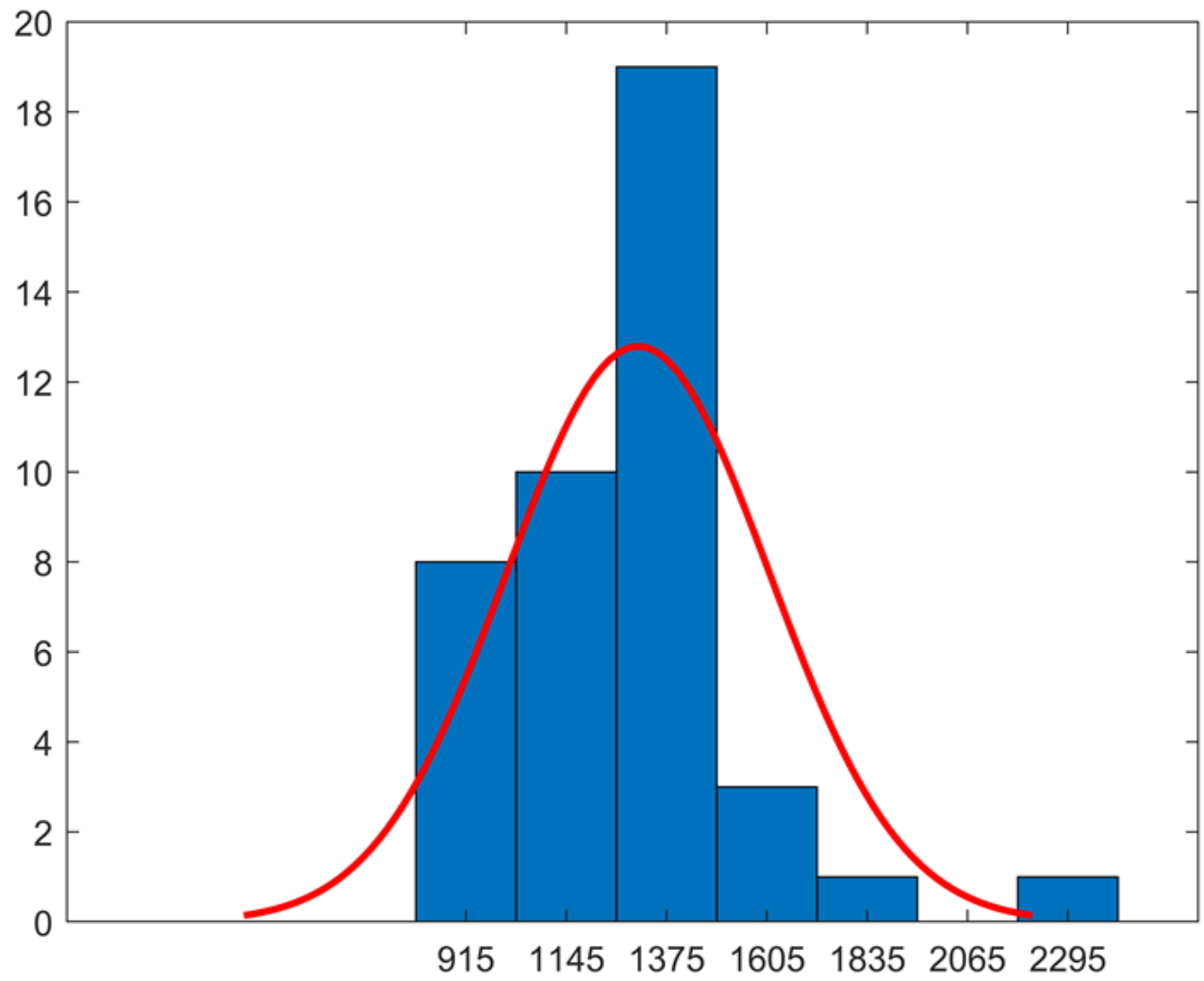

(A)

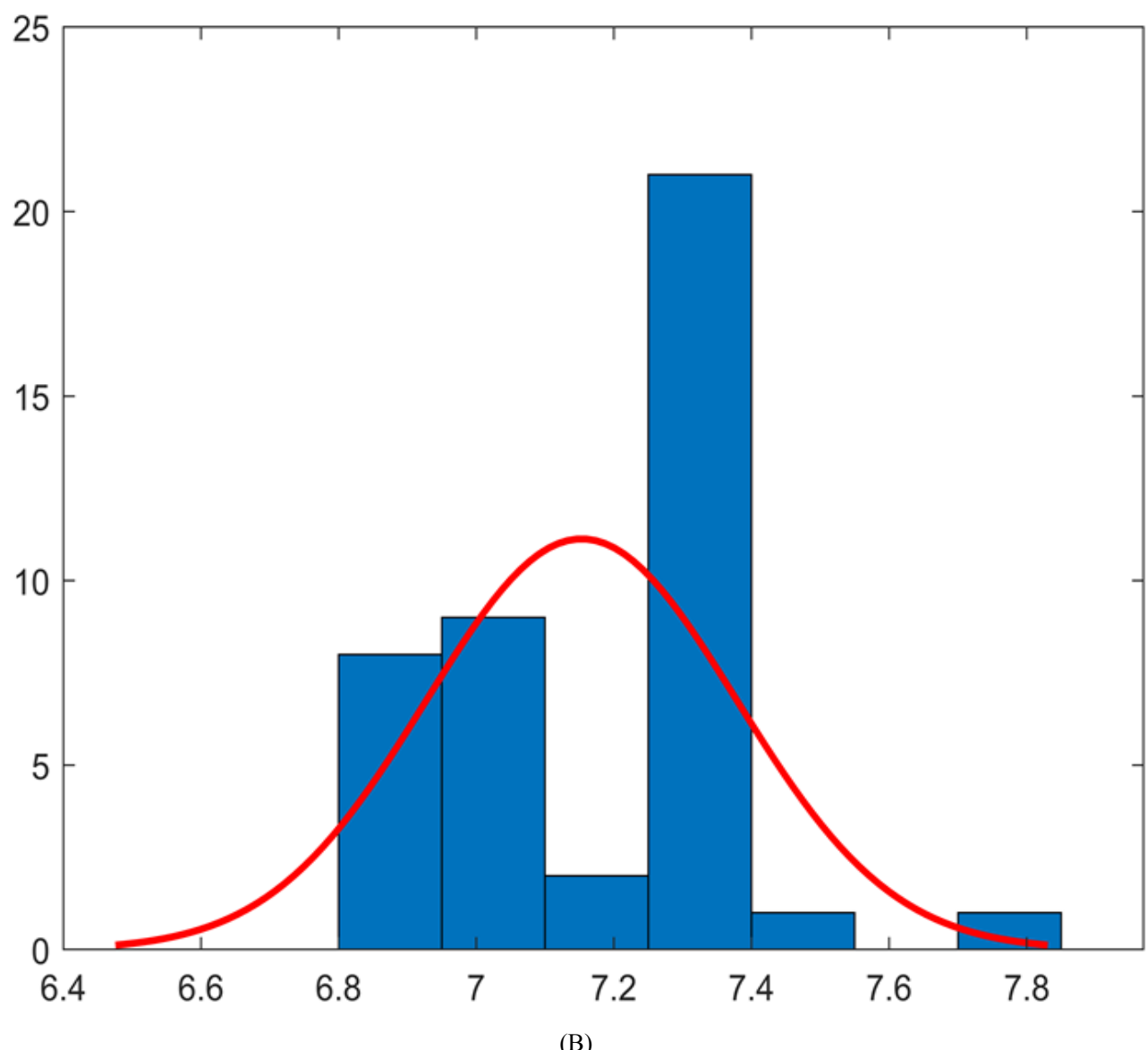

Figure 2. A (Untransformed) and B (Transformed) 


\subsection{The Effect of the Percentage of Training Datasets Size on the ANN Performance Metric}

A dedicated MATLAB script code was used to perform the required computations in this work. A back-propagation training algorithm was used in a feed-forward network trained with the LevenbergMarquardt algorithm.

The training, validation, and testing of ANN model for shear resistance prediction were conducted based on 1 hidden layer. These processes were carried out with 2 subset training dataset sizes $(70 \%$ and $90 \%$ randomly selected training datasets) and the experiment was carried out by varying the neuron number from 1 to 12 . The selection of 12 neurons was made by the reason of to simulate a simple ANN model by 1 to 3 number of neurons, which has large bias, to a moderately to super complex ANN model, which was represented by 4 to 12 neurons. Figure 3A to Figure $3 \mathrm{~B}$ show the plot of performance metric, RMSE, versus the increasing neuron number for each training, validation, and testing process of the network.

It can be observed that as the training datasets increase in size, the performance of ANN (RMSE) is apparently decreased by certain percentages. During this stage, the random parameters values (weight and bias) will be served as the input parameters to each of neuron of the hidden layer and the output layer to learn the nature of the data and to predict the output value in the output layer in each consecutive neuron number. The performance of ANN (RMSE) of each consecutive neuron was computed by updating the weight and bias value to obtain a more better ANN model. This process of network learning will be continuing for the first 12 neuron. As an example, when the neuron in the hidden layer is set to be 2 , the root mean square error increases from 0.4367 (70 \% training datasets) to 0.4884 ( $90 \%$ training datasets). This finding agrees with [30] that conclude that the network accuracy is improved as the number of training dataset is enough to represent the actual condition. The $70 \%$ of training dataset is found to be the optimum data division as compared to the $90 \%$ training datasets. The RMSE is greatly dependent on the type of activation function used and the number of hidden layers [42].

The optimal number of neurons in the hidden layer is decided by using the early-stopping algorithm. This algorithm is chosen as it was widely used especially in the neural networks starting from year of 1990 to study the relationship between the model complexity and the network performance. As shown in Figure 3A and Figure $3 \mathrm{~B}$, the RMSE curve reduces significantly from the first to the second neuron for each training validation and testing dataset. This is attributed to the network learning on the datasets as the training is in progress and agrees with other researchers' works [43]. However, the RMSE values suddenly rise when the neuron number is more than 2 , indicating that the ANN model starts to be in overfitting situation, which needs to be avoided. At this point, it is considered that the optimal number of neurons for this network is 2 . The usage of neuron number more than 2 will further increase the RMSE values for all training, validation, and testing datasets. These patterns of findings were in line with the work done by [43, 44]. The addition of more neurons in a first hidden layer has negative effect of ANN performances. This finding further supports the conclusion statement by Ćirović and Aleksendrić [45] that too many neurons in the first hidden layer are not desirable when training ANNs with LM algorithm.

\subsection{The ANN Performance under the Optimal Neuron on Predicting the Shear Resistance}

The optimal network performance is investigated based on the optimal neuron number, which is 2 , and under $90 \%$ of training datasets. The selection of this percentage of training dataset is decided by the lower RMSE value as $90 \%$ of the training datasets were employed during the network learning stage. Under the optimal network, the correlation value, $\mathrm{R}$, between the predicted and the actual shear resistance during training (0.96915), validation (0.89914) and testing (0.9648) were shown in Figure 4. The validation datasets showed relatively less correlated than the training and the testing datasets; showing its limitation of prediction based on the optimal ANN model on unseen datasets. A further study on solving this issue by feature selection techniques such as the Principal Component Analysis (PCA) on the existing datasets is to analyze the contribution of new transformed datasets once extracted by PCA.

On the other hand, Figure 5 shows the prediction of the optimal network based on the certain validation and testing dataset, which indicate excellence agreement with the actual shear resistance values. It can be inferred that the there was no difference of prediction between the testing datasets and the validation datasets from statistical point of view.

In this optimal network, the combination of $90 \%$ of the training dataset and the usage of 2 neuron in the hidden layer, will be the parameters that affecting the optimality of the error; the weights and bias of neurons inter-connection are adjusted during training stage until the network learns the correlation between input and output variables, i.e., until the minimum mean square error (MSE) is obtained. 


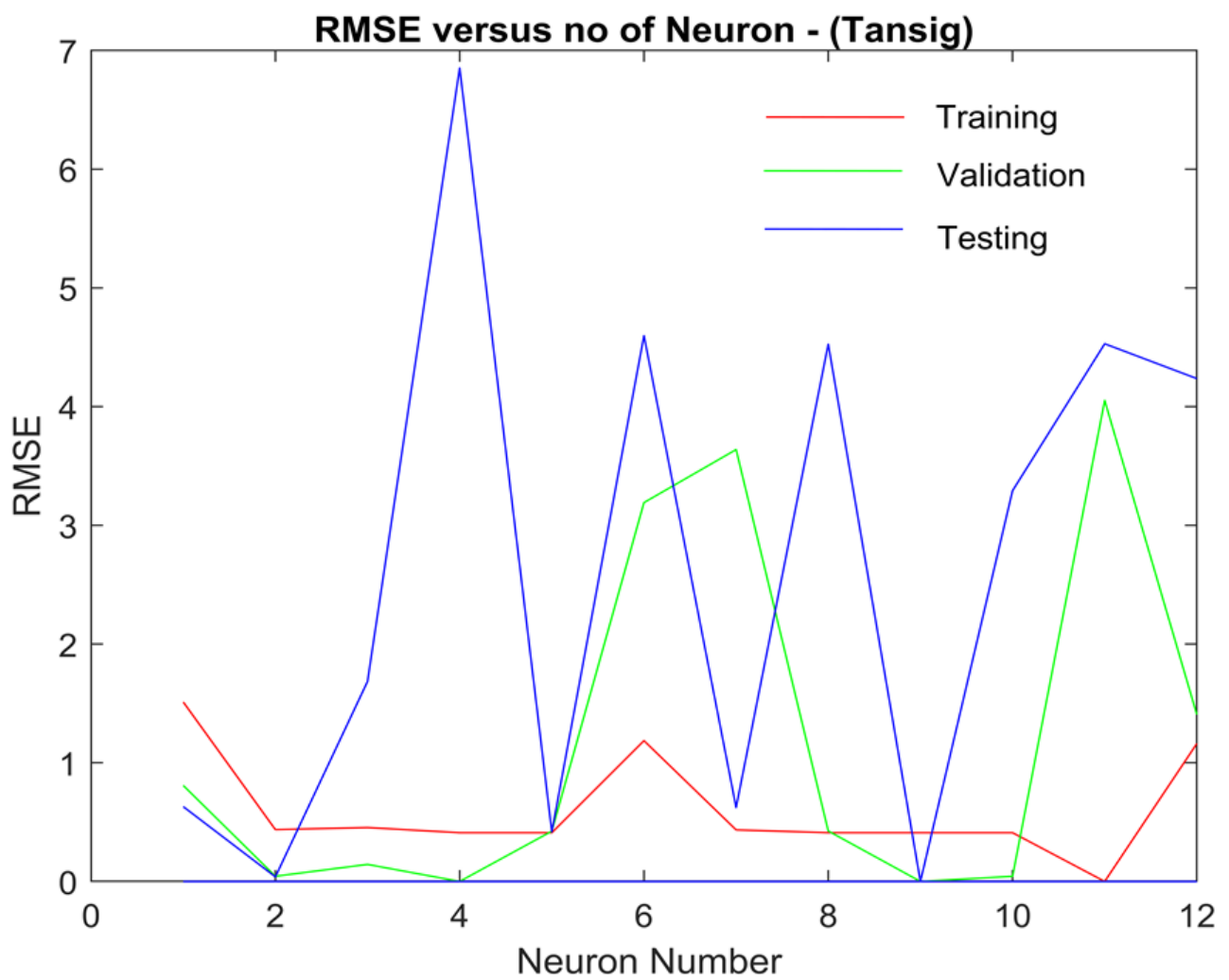

(A)

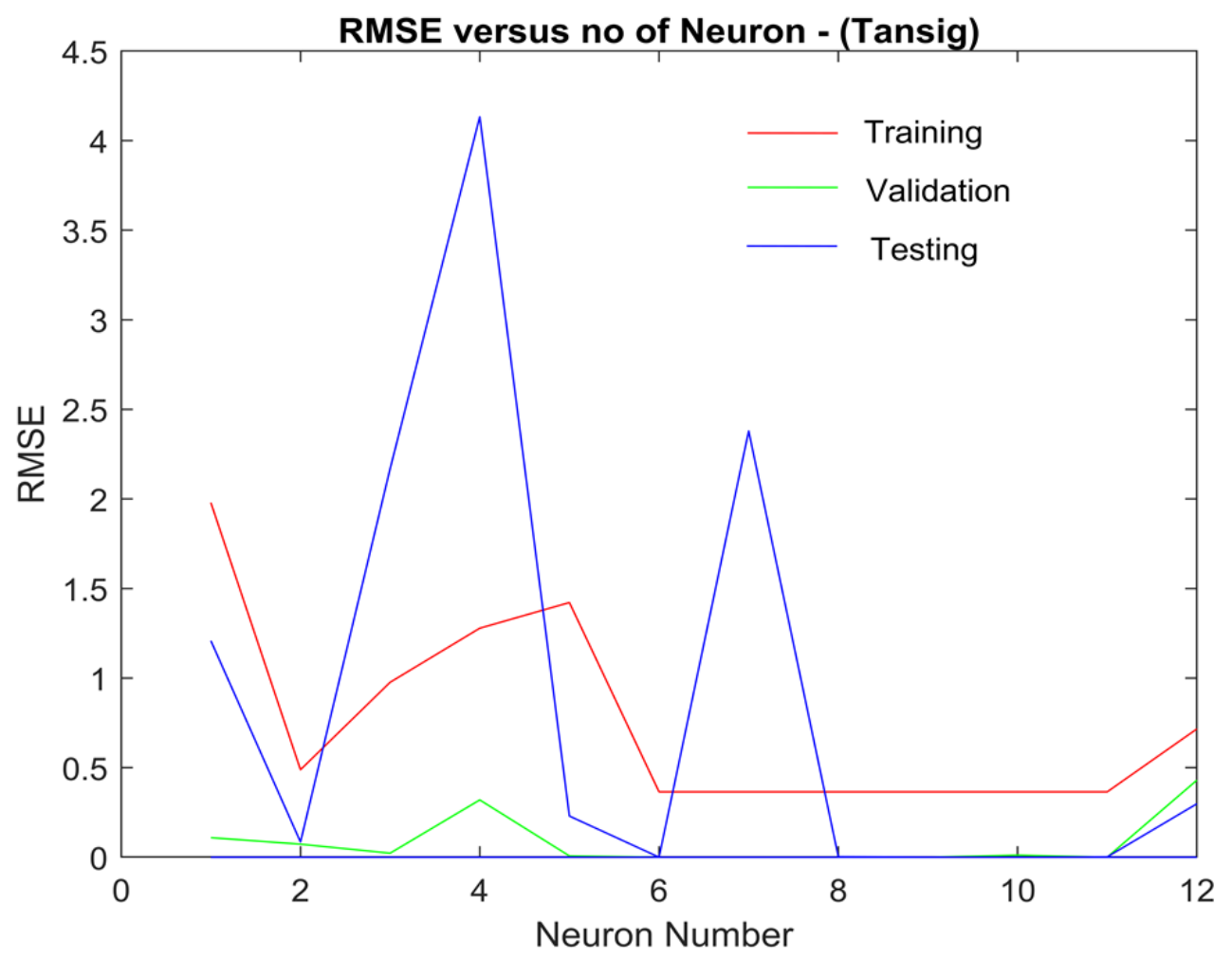

(B)

Figure 3. A (70\%) and B (90\%) 

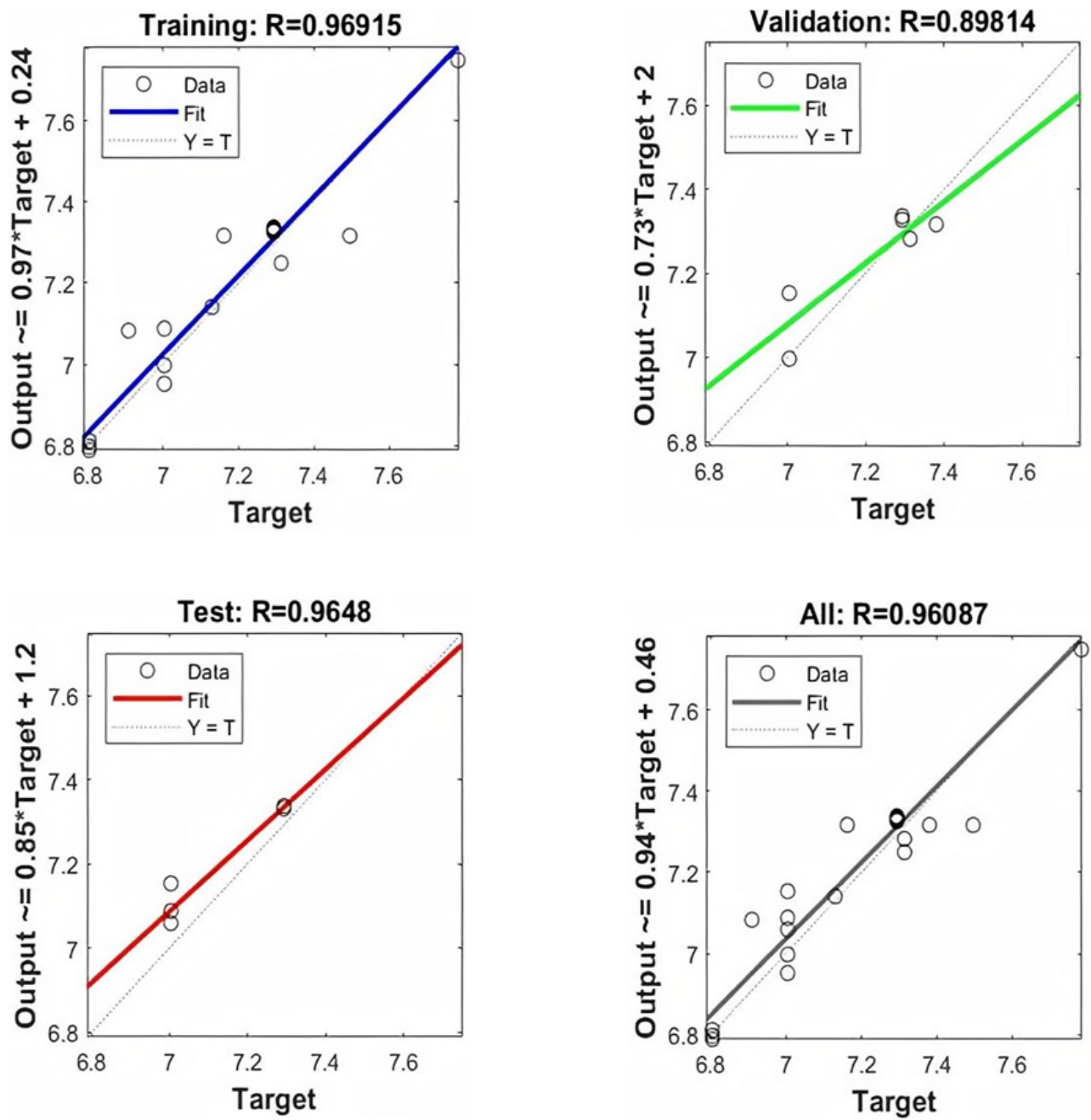

Figure 4. Performance of $\mathrm{R}$ between the predicted and shear resistance

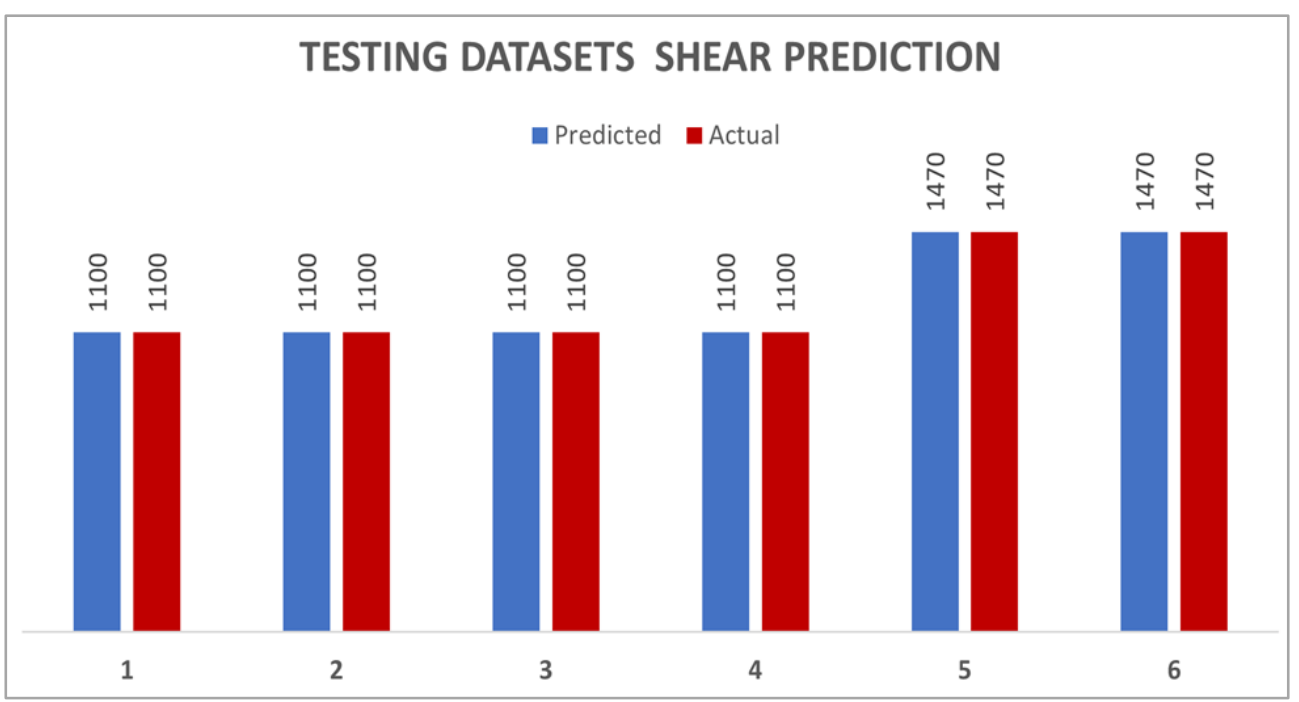




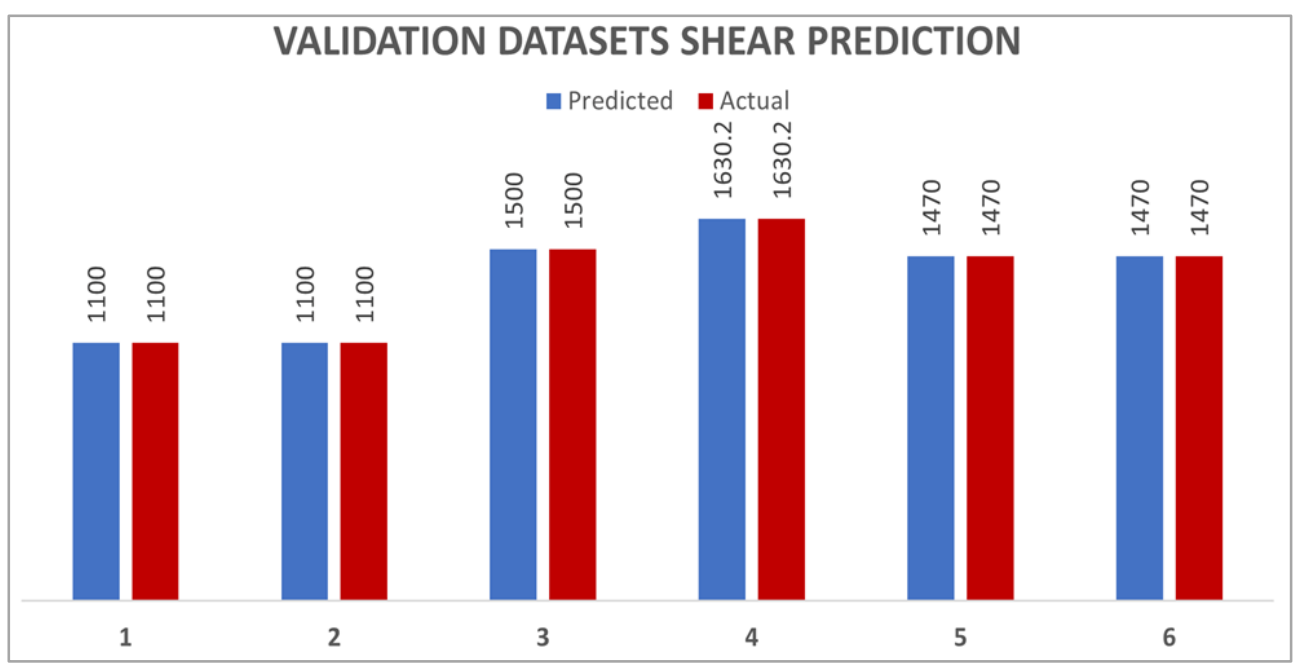

Figure 5. Prediction of optimal network under validation and testing data

\section{Conclusions}

The main contribution in this study is to provide an optimal network for predicting the shear resistance of SFRC by changing the testing set size and the number of neurons in the first hidden layer. The optimal neuron number on a single hidden layer is determined by the early stopping algorithm by fixing the training set size as $90 \%$. The result of this study is very useful for predicting the resistance of shear of fiber reinforced concrete material.

We highlight the following results of this study:

(i). The normalization of input vectors and the standardization of the output vectors are important to ensure better learning of the network to obtain the minimum error. The standardization of the untransformed output vector reduces the skewness value from 0.8442 to 0.0361 , which lessens the effect of extreme distribution of the shear resistance values and thus facilitates the learning stage of the network.

(ii). It was observed that by using $70 \%$ of the whole data as the training set size will produce the lowest RMSE. Training set size is one of the important hyperparameter in artificial neural network to be carefully selected to ensure the higher accuracy of shear resistance values prediction.

(iii). The optimal number of neurons of the network is found to be two, which was determined by using the early stopping algorithm. This optimal neuron number network can be used to perform the shear resistance predictions after new input dataset obtained from the validation (15\%) and verification datasets $(15 \%)$ used for prediction.

(iv). The optimal network with $70 \%$ training set size and 2 neuron number was used to perform the forecast of the shear resistance values. The optimal network perfectly predicts the shear resistance with all unseen data (testing and validations data), showing that RMSE of the network has been globally minimized.

(v). Further works on improving the limitation of the optimal ANN model to predict the unseen data (i.e., the coefficient of correlation of the validation datasets) by extracting the contributing outputs by using the Principal Component Analysis to the existing datasets. It is hypothesized that by using PCA, the unnecessary or non-contributing datasets shall be extracted out from the existing data.

\section{Acknowledgments}

The authors acknowledged Universiti Teknologi MARA Cawangan Pulau Pinang, Permatang Pauh Campus for the support of the provision of MATLAB software for this study.

\section{REFERENCES}

[1] L. Xuesen, D. Jie, D. Mingke. Shear Behavior of High Ductile Fiber Reinforced Concrete Beams, Alexandria Engineering Journal, Vol.60, No.1, 1665-1675.

[2] S. Mansor, R.N. Mohamed, N.A. Shukri, M.S.N. Mahmoor, N. Azilah, F. Zamri. Overview on the Theoretical Prediction of Shear Resistance of Steel Fibre in Reinforced Concrete Beam, IOP Conference Series: Earth and Environmental Science, doi:10.1088/1755-1315/220/1/012033, 1-10, 2019.

[3] J.H. Hwang, D.H. Lee, H. Ju, H. Kim, K.S. Seo, S.Y. Kang, J.W. Kang. Shear behavior models of steel fiber reinforced concrete beams modifying softened truss model approaches, Materials, Vol. 6, No. 10, 4847-4867.

[4] J.A.O. Barros, S.J. Foster. An Integrated Approach for Predicting the Shear Capacity of Fibre Reinforced Concrete 
Beams, Engineering Structures, Vol. 174, 346-357.

[5] Y.C. Choi, J.H. Moon, E.J. Lee, K.S. Park, K.S. Lee. Development of Shear Strength Equation for Beam-Column Connections in Reinforced Concrete and Steel Composite System, International Journal of Concrete Structures and Materials, Vol. 11, No. 2, 185-197.

[6] N. Spinella. Shear Strength of a Full-Scale Steel Fibre-Reinforced Concrete Beams without Stirrups, Computers and Structures, Vol. 11, No. 5, 365-382.

[7] A. Luigi, National Research Council, Guide for the Design and Construction of Fiber-Reinforced Concrete Structures, Advisory Committee on Technical Recommendations for Construction, CNR-DT 204/2006, 1-55

[8] G.B. Jumaa, A. R. Yousif. Predicting Shear Capacity of FRP-Concrete Beams without Stirrups by Artificial Neural Networks, Gene Expression Programming, and Regression Analysis, Advances in Civil Eng., Vol. 2018, 1-16.

[9] T. F. Awolusi, O.L. Oke, O. O. Akinkurolere, O.D. Atoyebi. Comparison of Response Surface Methodology and Hybrid-Training Approach of Artificial Neural Network in Modelling the Properties of Concrete Containing Steel Fibre Extracted from Waste Tyres, Cogent Engineering, Vol. 6.

[10] J.P. Maran, V. Sivakumar, K. Thirugnanasamdham, R. Sridhar. Artificial Neural Network and response surface methodology modelling in mass transfer parameters predictions during osmotic dehydration of Carica papaya L. Alexandria, Engineering Journal, 2013, Vol. 52, 507-516.

[11] K.G. Sheela, S.N. Deepa. Review on Methods to Fix Number of Hidden Neuron in Neural Networks, Mathematical Problems in Engineering, Vol. 2013, https://doi.org/10.1155/2013/425740.

[12] A.D. Gavrilov, A. Jordache, M. Vasdani, J. Deng. Preventing Model Overfitting and Underfitting in Convolutional Neural Networks, International Journal of Software Science and Computational Intelligence, Vol. 10, No. 4, 1-10.

[13] K.S. Hornik, M.H. White. Multilayer Feedforward Networks Are Universal Approximators, Neural Netw, Vol. $2,359-366$

[14] K.S. Hornik, M.H. White. Some New Results on Neural Networks Approximations, Neutral Netw, Vol. 6, 1069-1072.

[15] G.-B. Huang, H.A. Babri. Upper bounds on the number of hidden neurons in feedforward networks with arbitrary bounded nonlinear activation functions, IEEE, Vol. 9, No.1, 224-229.

[16] G. Du, L. Bu, Q. Hou, J. Zhou, B. Lu. Prediction of The Compressive Strength of High Performance Self-Compacting Concrete by an Ultrasonic-Rebound Method Based on a GA-BP Neural Network, PLOS One, https://doi.org/10.1371/journal.pone.0250795

[17] J.L. Liang, G.R. Jin, Z.X. Shen. Prediction of Shear Characteristics of Unsaturated Soil Based in BP Neural Network, Second International Conference on Civil Architecture and Energy Science (CAES 2020), EDP Sciences, 2020
[18] S. Kiran, B. Lal. Modelling of Soil Shear Strength Using Neural Network Approach, EJGE, Vol. 21, No. 2016, 3751-3771.

[19] A. Amin, S.J. Foster. Shear Strength of Steel Fibre Reinforced Concrete Beams with Stirrups, Engineering Structures, Vol. 111, 323-332.

[20] A. Amin, S.J. Foster, M. Watts. Modelling the tension stiffening effect in SFR-RC, Magazine of Concrete Research, Vol. 68, No. 7, 339-352.

[21] T. Greenough, M.L. Nehdi. Shear Behavior of Fiber-Reinforced Self-Consolidating Concrete Slender Beams, Materials Journal, Vol. 105, No. 5, 468-477.

[22] J. H. Hwang, D. H. Lee, H. Ju, K.S. Kim, S.Y. Seo, J.W Kang. Shear Behaviour Models for Steel Fibre Reinforced Concrete Beams, Modifying Softened Truss Model Approaches, Materials, Vol. 6, No. 10, 4847-4867.

[23] M. Imam, L. Vandewalle, F. Mortelmans, D.V. Gemert. Shear domain of fibre-reinforced high-strength concrete beams, Engineering Structures, Vol. 19, No. 9, 738-747.

[24] Y. Kwak, M.O. Eberhard, W. Kim, J. Kim. Shear Strength of Steel Fiber-Reinforced Concrete Beams without Stirrups, Structural Journal, Vol. 99, No. 4, 530-538.

[25] M. A. Qissab, M. M. Salman. Shear strength of non-prismatic steel fiber reinforced concrete beams without stirrups, Structural Engineering and Mechanics, Vol. 67, No. $4,347-358$

[26] B, Singh, K. Jain. Appraisal of steel fibers as minimum shear reinforcement in concrete beams, ACI Structural Journal, Vol. 111, No. 5, 1191-1202.

[27] M. Spinella. Shear strength of full-scale steel fibre-reinforced concrete beams without stirrups, Computers and Concrete, Vol. 11, No. 5, 365-382.

[28] M.R. Zarrinpour, S.H. Chao. Shear strength enhancement mechanisms of steel fiber-reinforced concrete slender beams, ACI Structural Journal, Vol. 114, No. 3, 729-742.

[29] C. Feng, H. Wang, N. Lu, T. Chen, H. He, Y. Lu, X.M. Tu. Log Transformation and its Implications for Data Analysis, Shanghai Archives of Psychiatry, Vol. 26, No. 2, 105-109.

[30] M.A. Shahin, H.R. Maier, M.B. Jaksa. Data Division for Developing Neural Networks Applied to Geotechnical Engineering, Journal of Computing in Civil Engineering, Vol. 18, No. 2, 105-114

[31] Y.S.T. Hong, Dynamic Nonlinear state-space model with a Neural Network via Improved Sequential Learning Algorithm for An Online Real-Time Hydrological Modelling, Journal of Hydrology, Vol. 2012, No. 468-469, $11-21$

[32] T. Rajaee, A. Shahabi. Evaluation of wavelet-GEP and wavelet-ANN hydrid models for prediction of total nitrogen concentration in coastal marine waters, Arab J. Geosci, Vol. 2016, No. 9.

[33] S. Areerachakul, P. Sophatsahit, C. Lursinap. Integration of unsupervised and supervised neural networks to predict dissolved oxygen concentration in canals, Ecol. Modell, Vol. 2013, No. 261-262, 1-7. 
[34] A.A.M. Ahmed. Prediction of dissolved oxygen in Surma River by biochemical oxygen demand and chemical oxygen demand using the artificial neural networks (ANNs), J. King Saudi Univ, Eng. Sci., Vol. 2017, No. 29, 151-158.

[35] M.J. Alizadeth, M.R. Kavianpour. Development of wavelet-ANN models to predict water quality parameters in Hilo Bay, Pacific Ocean, Mar. Pollution Bull., Vol. 2015, No. 98, 171-178.

[36] Y. Wang, J. Zhou, K. Chen, Y. Wang, L. Liu. Water quality prediction method based on LSTM neural network, In Proc. of the $201712^{\text {th }}$ International Conf. on Intelligent System and Knowledge Engineering, ISKE 2017, Nanjing China, 24-26 Nov. 2017.

[37] S. M. Cabaneros, J. K. Calautit, B.R. Hughes. A review of artificial neural network models for ambient air pollution prediction. Environ. Model. Softw. Vol. 2019, No. 119, 285-304.

[38] R. Barzegar, A. A. Moghaddam, J. Adamowski, B. Ozga-Zielinski, Multi-step water quality forecasting using a boosting ensemble multi-wavelet extreme learning machine model. Stoch. Environ. Res. Risk Assess, Vol. 2018, No. 32, 799-813.

[39] J. T. DeWeber, T. Wagner, A regional neural network ensemble for predicting mean daily river water temperature. J. Hydrol. Vol. 2014, No. 517, 187-200.
[40] Z. Cataltepe, Y.S. Abu-Mostafa, M. Magdon-Ismail, No Free Lunch for Early Stopping, Neural Computation, Vol. 11, No.4, 995-1009.

[41] D.K. Marcus, K. Mats. Salience and Skewness Preferences, European Economic Association, Vol. 18, No.5, 2057-2107.

[42] A. Serwa. Studying the Effect of Activation Function on Classification Accuracy Using Deep Artificial Neural Networks, Journal of Remote Sensing and GIS, Vol. 6, No. $3,1-6$.

[43] E.O. Ezugwu D.A. Fadare, J. Bonney, R.B. Da Silva, W.F. Sales. Modelling the correlation between cutting and process parameters in highspeed machining of Inconel 718 alloy using an artificial neural network, International Journal of Machine Tools and Manufacture, Vol. 45, No. 12-13, 1375-1385.

[44] A. Shickin, A. Buevich, A. Sergeev, E. Baglaeva, I. Subbotina, J. Vasiler, K.S. Maria. Training Alogorithm in Predicting of the Content of Chemical Elements in the Upper Soil Layer, Proc. of the 44-th Int. Conf. on Applications of Mathematics In Engineering and Economics, AIP Conf.Proc. 2048.

[45] V. Ćirović, d. Aleksendrić. Development of neural network model of disc brake operation, FME Transactions, Vol. 38, No. 1, 29-38. 